\title{
EVALUACIÓN DE LA LABOR DOCENTE EN LA PRÁCTICA IGUALITARIA DE GÉNERO EN EDUCACIÓN INICIAL
}

\author{
Pozo Andrea ${ }^{1}$, García Ruth ${ }^{2}$, Casa Erika ${ }^{3}$ y Logroño Moisés ${ }^{4}$ \\ elizandy-ep@hotmail.com ${ }^{1}$, ruth0277@hotmail.com², ms.educacion81inicial@gmail.com³ \\ moiseslogrono@yahoo.com ${ }^{4}$ \\ https://orcid.org/0000-0003-0037-69751, https://orcid.org/0000-0002-0044-2488 ${ }^{2}$ \\ https://orcid.org/0000-0001-7562-1678 ${ }^{3}$, https://orcid.org/0000-0002-3759-3896 ${ }^{4}$ \\ Universidad Nacional de Rosario ${ }^{1},{ }^{2,3}$, Universidad Central del Ecuador ${ }^{4}$ \\ Quito-Ecuador
}

Recibido (01/07/20), Aceptado (17/07/20)

\begin{abstract}
Resumen: La inequidad de género en el ámbito educativo se puede considera como un problema social que afecta a nivel mundial. El objetivo de esta investigación fue analizar la práctica docente en el ámbito de la educación inicial, considerando la influencia de los familiares y docentes en los primeros años de vida del infante donde se atribuye características estereotipadas de comportamiento adjuntas a un género. La metodología se basó en la investigación cualitativa y cuantitativa, con una muestra total conformada por 119 estudiantes del nivel inicial y 6 docentes de cuatro instituciones fiscales del Distrito 9, Parroquia El Quinche, El instrumento utilizado fue una lista de cotejo para los infantes y un cuestionario a los docentes. El estudio revelo que las docentes no generan la igualdad de género en las aulas, existe un desconocimiento de este tema y también señala que los factores culturales del entorno son fundamentales en este proceso.
\end{abstract}

Palabras Clave: Equidad de género, factores culturales, estereotipos, educación inicial.

\section{EVALUATION OF TEACHING WORK IN GENDER EQUAL PRACTICE IN INITIAL EDUCATION}

\begin{abstract}
Gender inequality in education can be seen as a global social problem. The objective of this research was to analyze teaching practice in the field of initial education, considering the influence of family members and teachers in the infant's early life where stereotypical behavioral characteristics attached to a gender are attributed. The methodology was based on qualitative and quantitative research, with a total sample of 119 students from the initial level and 6 teachers from four public institutions from District 9 , el Quinche Town. For infants, the instrument used was a checklist and a questionnaire for teachers. The study revealed that teachers do not generate gender equality in classrooms, there is an ignorance of this topic, and also it pointed out that the cultural factors of the environment are fundamental in this process.
\end{abstract}

Keywords: Gender equity, cultural factors, stereotypes, initial education. 


\section{I.INTRODUCCIÓN}

La evaluación de la práctica docente es un espacio socializador, encargada de que hombres y mujeres asimilen las formas de actuar esperadas y aprobadas socialmente, esta labor atraviesa por cuestiones institucionales, ideológicas y epistemológicas. [1]. En este contexto la evaluación de la práctica docente da a conocer las formas de hacer de los profesores y brinda aportes en las mejoras de la formación inicial o permanente de los mismos como factor clave de la calidad educativa [2].

El género es una categoría social [3], históricamente a partir de la estructura corporal biológica, sexo con el cual se nace, donde la sociedad ha asignado a los individuos el rol de hombre o mujer y en base a ello las características, actitudes y comportamientos que se espera según su sexo.

La educación inicial prioriza la importancia de los primeros años de vida del infante, el entorno donde se desenvuelve es vital, aquí los niños y niñas se convierten en constructores activos de su propio mundo social [4], un universo que reproduce las características definitorias del marco adulto que les rodea. Es así que desde este aspecto es relevante analizar y evaluar las concepciones y percepciones de equidad de género desde este escenario, reflexionando el rol que tiene la práctica educativa en la producción y transmisión de una igualdad de género que se dan en cosas tan simples como el uso de lenguaje solo en masculino tanto a niños como a niñas, estereotipos que encierran a las niñas y niños en moldes femeninos y masculinos, los juegos que se realizan en los patios, el uso de material con contenido sexista y androcéntrico de manera intencional o no, a pesar de que en la Constitución del Ecuador [5] se mencione la igualdad de oportunidades para hombres y mujeres. Diversos estudios han expuesto que en la práctica pedagógica se reproducen esquemas identitarios discriminadores [6].

La investigación tuvo como objetivo analizar la práctica docente en el ámbito de la educación inicial, considerando la influencia de los familiares y docentes en los primeros años de vida del infante [7]. Donde se atribuye características estereotipadas de comportamiento adjuntas a un género femenino o masculino, tales como vestimentas, comportamientos, actitudes, juegos, tareas, obligaciones entre [8].

La idea que antecedente, incide en la reproducción de roles de género en la edad adulta de una manera equivocada, este trabajo pretende dar a conocer que los docentes de educación inicial deben buscar estrategias, actividades, juegos, tareas que permitan una equidad de género tanto a niños como a niñas, y no estereotipar su manera de concebir sus roles de género en la sociedad.
La metodología empleada en este trabajo se basó en la investigación cualitativo y cuantitativo, de tipo descriptivo y exploratorio, mediante la revisión de la información a través de la exploración bibliográfica se posibilitó inferir las aportaciones teóricas y conceptuales de diversos autores, la aplica $\neg$ ción de entrevistas y cuestionario, se aplico el método de la triangulación para la elaboración de las conclusiones y las recomendaciones.

En las conclusiones preliminares, se puede comentar que las estrategias didácticas deben fomentar la participación de manera igualitaria a niños como a niñas; otro factor que se observo fue que él personal docente no cuenta con materiales para trabajar el tema de género, además este personal tampoco siente motivación por la elaboración del mismo.

Este trabajo se divide en cuatro secciones, la primera parte consiste en la descripción del problema, la segunda el desarrollo que sustenta la investigación, la tercera presenta la metodología que se realizó para llegar a la sección cuarta donde se expone los resultados.

\section{II.DESARROLLO}

Al incorporarse las mujeres al campo laboral y ampliar la cobertura de la educación inicial, recae la responsabilidad de la formación integral de estos niños y niñas en las instituciones educativas [9]. El papel que los establecimientos formativos realizan en este acompañamiento es decisivo para la construcción integral del ser humano, es el docente quién se convierte en la persona que pasa la mayor parte de tiempo [10], aportando en el desarrollo de las capacidades cognitivas, emocionales y sociales de los niños y niñas. La educación puede constituirse en un vehículo que genere iniquidades de género [9], conviene modificar la interacción que se generan a lo interno del aula, donde se establezcan ambientes caracterizados por la equidad.

El tema de género apunta a una sociedad más justa [9], favoreciendo la oportunidad de igualdades para hombres y mujeres "inédito" [11]. El termino, es otorgado por John Money en 1995, para anunciar los aspectos simbólicos que socialmente se asignan a hombres y mujeres [9], su concepto inicia alrededor de los años sesenta, para algunos autores, el género determina una construcción social de lo femenino y lo masculino, manifestándose en un conjunto de conductas aprendidas culturalmente de acuerdo a los procesos históricos y lugares en que su vida se desarrolla, como la escuela, los miembros de la familia, la iglesia, los medios de comunicación inédito [11], siendo estos espacios de socialización.

Es la educación quien promueve un plan de acción pedagógico, de formación, crítico, dinámico, partici- 
pativo y creativo, encaminado a crear experiencias e aprendizajes que provoquen una aproximación entre el conocimiento, la realidad y la producción de significado del sujeto educativo, ampliando una serie de saberes y competencias que van incidiendo sobre su identidad personal y ciudadana [12], Al hacer alusión al sujeto, Morín, propone que la educación no solo aporte al desarrollo de las capacidades cognitivas [13], al mismo tiempo se debe cooperar a la construcción de habilidades relacionadas con la ciudadanía tendiente a consolidar una subjetividad que permita la transformación de la realidad de cambios paradigmáticos que consideren la contextualización de integración de los saberes.

Al hablar de la integración de los saberes nos referimos a las características socio-culturales e históricas [13], que determinan como hombres y mujeres piensan, sienten, interactúan y dividen sus funciones, que hace referencia a la categoría género y sexo inédito [11], a partir de sus diferencias biológicas de sexo, que son naturales, congénitas y universales. Por fines de análisis se separó los elementos biológicos y sociales, en la actualidad también los aspectos sexuales/biológicos se han ido condicionando socialmente en sus modos de realización y expresión inédito [11], que no son analizados en este trabajo. Es necesario conocer que estos dos términos están íntimamente relacionados, sin embargo, estos no son iguales.

Los roles de género son el conjunto de papeles y funciones que conforman el modelo preestablecido de ser hombre o ser mujer en una sociedad determinada [14]. Los niños aprenden a ocupar los espacios sociales, materiales y simbólicos reservados a lo propio de un hombre. Las niñas aprenden a ocupar los espacios sociales, materiales y simbólicos reservados a lo propio de una mujer. Los roles de género son cambiantes y culturalmente específicos. El problema no es la diferencia de roles sino su jerarquización y el modo en que se socializan inédito [11].

En el proceso de la socialización se transfieren, mantienen y reproducen valores, costumbres, típicos de una cultura, en un contexto social determinado, puede ser formal y no formal de manera intencional o no inédito [11], estos se transmiten a partir de la primera infancia y continúan a lo largo de su vida, el hombre está hecho para el éxito, la dirección y la política mientras que la mujer está hecha al trabajo doméstico, vivir el éxito de otros, generalmente del esposo. Este proceso tiene varios agentes como la familia, que posee un importante papel en la primera etapa de su vida, son los primeros modelos que el niño y la niña va a tener "inédito" [11], el segundo es el sistema educativo donde se refleja la estructura social de valores y creencias de una determinada cultura, este agente puede llegar a ser un importante instrumento de cambio mediante el currículo oculto y los procesos de interacción social que se da [12].Por último los medios de comunicación que responden a los requerimientos de la audiencia ellos reproducen los valores culturales e ideales existentes sobre el género inédito [11]. Aunque estos agentes contribuyen muchas veces al mantenimiento de los estereotipos, también pueden ser agentes de cambio de las desigualdades inédito [11].

Es prescindible mencionar que esta investigación se realizó en el contexto rural, donde el sistema escolar de las niñas es desfavorable inédito [11], las estadísticas que recogen la situación de las mujeres de 15 años y más reflejan trayectorias de infancias marginales con respecto a la escuela, especialmente en las etapas del periodo preescolar donde se suman los factores endógenos, culturales y de género inédito [11]. Para hablar de masculino y femenino en estas sociedades, es necesario considerar los aspectos y las problemáticas políticas, económicas y territoriales específicas que afrontan los pueblos y comunidades.

Respecto a la consideración de los estereotipos de género INMUJERES inédito [11], estos reflejan las creencias populares sobre las actividades, atribuidos en función del género, las mujeres cuidan a las hijas e hijos, hacen comida y los hombres realizan los trabajos mecánicos, cargan las cosas pesadas. Así, el varón es educado para ser un buen proveedor y con derechos sobre los demás, ella, por su parte, para obedecer y depender de él. Estos estereotipos provocan que en ambos sean atrofiados o mutiladas potencialidades de su desarrollo integral "inédito" [11].

Para la psicología social y las teorías acerca de la motivación mencionan que los estereotipos de género pueden influenciar significativamente los comportamientos, tanto de profesores, como

de niñas y niños, afectando su desempeño y aprendizaje [14], uno que tiene alta aceptación en el mundo es, las niñas no son buenas para la matemática. Una vez que se establecen, los estereotipos son resistentes al cambio, así las personas se enfrenten a nueva información que pueda ser contradictoria con estos [14], si se desea conocer los estereotipos de los estudiantes, es primordial conocer también los de los adultos más relevantes en su vida, como son los padres y profesores [14]. Para todas las culturas, el proceso de la concepción de género entre los niños se acontece tempranamente y suele ser más destacado que las categorías de raza, de etnia, de religión o de nacionalidad [14]

Resulto necesario analizar la cotidianidad escolar, como analizar el lenguaje que el docente utiliza, como 
escribió Vygotsky, el significado de las palabras representa la forma más elemental de la unión entre el pensamiento y la palabra inédito [11], se puede constatar que el lenguaje hablado es el medio a través del cual se produce gran parte de la enseñanza y que el discurso docente incluye su forma de pensar inédito [11]. En la misma línea, rescatando la importancia del contexto en el que se desenvuelve el niño, Ausubel, menciona el aprendizaje significativo se da cuando el niño construye nuevos conocimientos sobre la base de una idea general que ya esté en su estructura mental, o conocimientos previamente adquirido, implica que la docente esté familiarizada con la historia personal, la diversidad cultural, la lengua, los saberes ancestrales, intereses y necesidades de los niños que constituyen elementos fundamentales inédito [11].

Al tener conciencia sobre el tema de género por parte de los y las docentes [15], se contribuye a que no se reproduzca prácticas sexistas que invisibilicen a las mujeres y la coloquen en una posición inferior a la del hombre [16], la educación con una perspectiva de género aporta en la formación de una nueva personalidad "inédito" [11], [17]. Mientras la escuela promueva cambios reales en las condiciones de vida de los infantes e igualar sus oportunidades [10], estará cooperando con su grano de arena a la equidad de género.

\section{III.METODOLOGÍA}

En este trabajo la muestra es de tipo no probabilístico intencional y está formada por 119 estudiantes del nivel inicial $(95,2 \%)$ y 6 docentes que constituyen el $4,8 \%$ de cuatro instituciones educativas fiscales del Distrito 9, Parroquia El Quinche ciudad de Quito. No se aplicó la fórmula de cálculo de la muestra ya que se consideró que es un número manejable (Institución $1=24 \%$, Institución $2=8.8 \%$; Institución $3=40 \%$ e Institución $4=$ $22 \%$. De docentes (Institución 1=1.6\%, Institución $2=$ $0.8 \%$; Institución $3=1.6 \%$ e Institución $4=0.8 \%$ ). De los cuales el $54,84 \%$ son hombres y el $45,16 \%$ mujeres.

El instrumento utilizado fue una lista de cotejo, a los niños y niñas para obtener datos e información durante las clases de las docentes [4], el instrumento se conformó por 11 ítems, en una escala de 2 alternativas de respuesta (si y no) conformada de 5 factores. El primer factor estuvo relacionado con el enfoque de género su relación y los roles. El segundo factor consto el género y sus principales agentes socializadores en la ruralidad. El tercer factor trato de los estereotipos de género. El cuarto factor se relacionó del currículo y las orientaciones metodológicas. En el quinto factor estuvo a fin a la educación inicial y la función de la docente como mediadora. También se utilizó un cuestionario con preguntas cerradas conformadas de 5 factores.

Para recopilar la información se coordinó con las autoridades de las instituciones educativas que participaron en el trabajo. Se aplicaron las técnicas de investigación con el consentimiento de los docentes, padres y madres de familia. Así mismo se informó a los participantes de la finalidad de la investigación y de la reserva de la información.

\section{IV.RESULTADOS}

El resultado de la lista de cotejo mediante la observación aplicada a los estudiantes, en las preguntas relacionadas al género, el $85,29 \%$ de los niños son los que notoriamente participan en las actividades que las docentes realizan, mientras las niñas con un 21, 56\%, según estos datos se argumenta que los niños son más participativos en las aulas de clases. En el ámbito de los juegos un $89,70 \%$ de los niños observados juegan con carritos, al futbol y al papá, solo el $10,30 \%$ ejecutan otros juegos. Mientras que un $94,11 \%$ de las niñas juegan con muñecas, a la cocinita y a la mamá, el 5,89\% juega a otra cosa, según los datos arrojados se puede concluir que se siguen desarrollando juegos que han sido tradicionalmente asignados para niños y otros para niñas, produciendo situaciones de machismo y una inequidad de género. A la hora que los estudiantes tienen que escoger un color de material un $98,52 \%$ de niños presentan rechazo a utilizar materiales de color rosado y el $1,48 \%$ no da importancia al color. Supuestamente colores que son solo para niñas impuestos por la sociedad [14].

Al procesar y analizar la información del cuestionario aplicado a las docentes, se llevó todo a tablas para comparar los resultados; a continuación, se presentan algunas tablas con resultados representativos a preguntas desarrolladas en el cuestionario. 
Tabla I. Termino masculino para designar a mujeres y varones

\begin{tabular}{|c|c|c|c|c|c|c|}
\hline $\begin{array}{c}\text { INS TITUCIONES } \\
\text { EDUCATIVAS } \\
\end{array}$ & CATEGORIA & FRECUENCIA & $\begin{array}{l}\% \\
\mathrm{~S}\end{array}$ & $\begin{array}{r}\% \\
\mathrm{CS} \\
\end{array}$ & $\begin{array}{r}\% \\
\text { AV } \\
\end{array}$ & $\begin{array}{l}\mathbf{\%} \\
\mathrm{N} \\
\end{array}$ \\
\hline \multirow[t]{4}{*}{27 DE FEBRERO } & S IEMPRE & 2 & \multirow[t]{4}{*}{$00 \%$} & \multirow[t]{4}{*}{$00 \%$} & \multirow[t]{4}{*}{$00 \%$} & \multirow[t]{4}{*}{$33,3 \%$} \\
\hline & CAS I S IEMPRE & 0 & & & & \\
\hline & A VECES & 0 & & & & \\
\hline & NUNCA & 0 & & & & \\
\hline \multirow{4}{*}{$\begin{array}{c}\text { JULIO M. } \\
\text { MATOVELLE }\end{array}$} & S IEMPRE & 1 & \multirow[t]{4}{*}{$00 \%$} & \multirow[t]{4}{*}{$00 \%$} & \multirow[t]{4}{*}{$00 \%$} & \multirow[t]{4}{*}{$16,7 \%$} \\
\hline & CAS I S IEMPRE & 0 & & & & \\
\hline & A VECES & 0 & & & & \\
\hline & NUNCA & 0 & & & & \\
\hline \multirow{4}{*}{$\begin{array}{c}\text { BELLO } \\
\text { HORIZONTE }\end{array}$} & S IEMPRE & 1 & \multirow[t]{4}{*}{$16,7 \%$} & \multirow[t]{4}{*}{$00 \%$} & \multirow[t]{4}{*}{$00 \%$} & \multirow[t]{4}{*}{$00 \%$} \\
\hline & CAS I S IEMPRE & 0 & & & & \\
\hline & A VECES & 0 & & & & \\
\hline & NUNCA & 0 & & & & \\
\hline \multirow[t]{4}{*}{ RICARDO ORTIZ } & S IEMPRE & 2 & \multirow[t]{4}{*}{$00 \%$} & \multirow[t]{4}{*}{$00 \%$} & \multirow[t]{4}{*}{$00 \%$} & \multirow[t]{4}{*}{$33,3 \%$} \\
\hline & CAS I S IEMPRE & 0 & & & & \\
\hline & A VECES & 0 & & & & \\
\hline & NUNCA & 0 & & & & \\
\hline \multicolumn{2}{|c|}{ TOTAL } & 6 & $16,7 \%$ & $00 \%$ & $00 \%$ & $83,3 \%$ \\
\hline
\end{tabular}

Fuente: Instituciones Distrito 9. Elaborada por los autores

En la Tabla I, se evidencio que las docentes en la práctica diaria siguen utilizando el genérico masculino para designar a mujeres y varones, este por más insignificante que pueda llegar hacer ayuda a promover la inequi- dad de género, también reflejo que existe un porcentaje mínimo que si utiliza en su lenguaje el termino asignado para niños y niñas.

Tabla II. Reproducción de juegos con estereotipo de género aprendidos en su hogar

\begin{tabular}{|c|c|c|c|c|c|c|}
\hline $\begin{array}{c}\text { INS TITUCIONES } \\
\text { EDUCATIVAS }\end{array}$ & CATEGORIA & FRECUENCIA & $\begin{array}{l}\% \\
\mathrm{~S}\end{array}$ & $\begin{array}{l}\% \\
\text { CS }\end{array}$ & $\begin{array}{c}\% \\
\text { AV }\end{array}$ & $\begin{array}{l}\% \\
\mathrm{~N}\end{array}$ \\
\hline \multirow{4}{*}{27 DE FEBRERO } & SIEMPRE & 2 & \multirow[t]{4}{*}{$33,3 \%$} & \multirow[t]{4}{*}{$00 \%$} & \multirow{4}{*}{$00 \%$} & \multirow{4}{*}{$00 \%$} \\
\hline & CAS I S IEMPRE & 0 & & & & \\
\hline & A VECES & 0 & & & & \\
\hline & NUNCA & 0 & & & & \\
\hline \multirow{4}{*}{$\begin{array}{c}\text { JULIO M. } \\
\text { MATOVELLE }\end{array}$} & SIEMPRE & 1 & \multirow[t]{4}{*}{$16,7 \%$} & \multirow[t]{4}{*}{$00 \%$} & \multirow[t]{4}{*}{$00 \%$} & \multirow[t]{4}{*}{$00 \%$} \\
\hline & CAS I S IEMPRE & 0 & & & & \\
\hline & A VECES & 0 & & & & \\
\hline & NUNCA & 0 & & & & \\
\hline \multirow{4}{*}{$\begin{array}{c}\text { BELLO } \\
\text { HORIZONTE }\end{array}$} & SIEMPRE & 1 & \multirow[t]{4}{*}{$16,7 \%$} & \multirow[t]{4}{*}{$00 \%$} & \multirow[t]{4}{*}{$00 \%$} & \multirow[t]{4}{*}{$00 \%$} \\
\hline & CAS I S IEMPRE & 0 & & & & \\
\hline & A VECES & 0 & & & & \\
\hline & NUNCA & 0 & & & & \\
\hline \multirow[t]{4}{*}{ RICARDO ORTIZ } & SIEMPRE & 2 & \multirow[t]{4}{*}{$33,3 \%$} & \multirow[t]{4}{*}{$00 \%$} & \multirow[t]{4}{*}{$00 \%$} & \multirow[t]{4}{*}{$00 \%$} \\
\hline & CAS I S IEMPRE & 0 & & & & \\
\hline & A VECES & 0 & & & & \\
\hline & NUNCA & 0 & & & & \\
\hline \multicolumn{2}{|c|}{ TOTAL } & 6 & $100 \%$ & $00 \%$ & $00 \%$ & $00 \%$ \\
\hline
\end{tabular}

Fuente: Instituciones Distrito 9. Elaborada por los autores

En la Tabla II, se observa que los juegos que son aprendidos desde sus hogares traen consigo estereotipos de juegos, para ellos es muy común está reproducción, es lo que están viviendo a diario en sus familias. 
Tabla III. Estrategias Metodológicas que se utilicen referente a género

\begin{tabular}{|c|c|c|c|c|c|c|}
\hline $\begin{array}{l}\text { INSTITUCIONES } \\
\text { EDUCATIVAS }\end{array}$ & CATEGORIA & FRECUENCIA & $\begin{array}{l}\% \\
\mathrm{~S}\end{array}$ & $\begin{array}{l}\% \\
\mathrm{CS}\end{array}$ & $\begin{array}{c}\% \\
\mathrm{AV}\end{array}$ & $\begin{array}{l}\% \\
\mathrm{~N}\end{array}$ \\
\hline \multirow{4}{*}{27 DE FEBRERO } & SIEMPRE & 0 & \multirow{4}{*}{$00 \%$} & \multirow[t]{4}{*}{$00 \%$} & \multirow{4}{*}{$00 \%$} & \multirow{4}{*}{$33,3 \%$} \\
\hline & CASI SIEMPRE & 0 & & & & \\
\hline & A VECES & 0 & & & & \\
\hline & NUNCA & 2 & & & & \\
\hline \multirow{4}{*}{$\begin{array}{l}\text { JULIO M. } \\
\text { MATOVELLE }\end{array}$} & SIEMPRE & 0 & \multirow[t]{4}{*}{$00 \%$} & \multirow[t]{4}{*}{$00 \%$} & \multirow[t]{4}{*}{$00 \%$} & \multirow[t]{4}{*}{$16,7 \%$} \\
\hline & CASI SIEMPRE & 0 & & & & \\
\hline & A VECES & 0 & & & & \\
\hline & NUNCA & 1 & & & & \\
\hline \multirow{4}{*}{$\begin{array}{c}\text { BELLO } \\
\text { HORIZONTE }\end{array}$} & SIEMPRE & 0 & \multirow[t]{4}{*}{$00 \%$} & \multirow[t]{4}{*}{$00 \%$} & \multirow[t]{4}{*}{$00 \%$} & \multirow[t]{4}{*}{$16,7 \%$} \\
\hline & CASI SIEMPRE & 0 & & & & \\
\hline & A VECES & 0 & & & & \\
\hline & NUNCA & 1 & & & & \\
\hline \multirow{4}{*}{ RICARDO ORTIZ } & SIEMPRE & 0 & \multirow[t]{4}{*}{$00 \%$} & \multirow[t]{4}{*}{$00 \%$} & \multirow[t]{4}{*}{$00 \%$} & \multirow[t]{4}{*}{$33,3 \%$} \\
\hline & CASI SIEMPRE & 0 & & & & \\
\hline & A VECES & 0 & & & & \\
\hline & NUNCA & 2 & & & & \\
\hline \multicolumn{2}{|c|}{ TOTAL } & 6 & $00 \%$ & $00 \%$ & $00 \%$ & $100 \%$ \\
\hline
\end{tabular}

Fuente: Instituciones Distrito 9. Elaborada por los autores

En la Tabla III, se analizó si las docentes utilizaban alguna estrategia metodológica para el tema de género, este reflejo que no, considero que se debe implementar una guía donde se pueda orientar con actividades y ta- lleres relacionados con género de esta manera estaríamos hablando de una igualdad de género para niños y niñas ofreciéndoles las mismas oportunidades.

Tabla IV. Material de apoyo para el trabajo de género

\begin{tabular}{|c|c|c|c|c|c|c|}
\hline $\begin{array}{l}\text { INSTITUCIONES } \\
\text { EDUCATIVAS }\end{array}$ & CATEGORIA & FRECUENCIA & $\begin{array}{l}\% \\
\mathrm{~S}\end{array}$ & $\begin{array}{l}\% \\
\text { CS }\end{array}$ & $\begin{array}{c}\% \\
\mathrm{AV}\end{array}$ & $\begin{array}{l}\% \\
\mathrm{~N}\end{array}$ \\
\hline \multirow{4}{*}{27 DE FEBRERO } & SIEMPRE & 0 & \multirow[t]{4}{*}{$00 \%$} & \multirow[t]{4}{*}{$00 \%$} & \multirow[t]{4}{*}{$00 \%$} & \multirow[t]{4}{*}{$33,3 \%$} \\
\hline & CASI SIEMPRE & 0 & & & & \\
\hline & A VECES & 0 & & & & \\
\hline & NUNCA & 2 & & & & \\
\hline \multirow{4}{*}{$\begin{array}{c}\text { JULIO M. } \\
\text { MATOVELLE }\end{array}$} & SIEMPRE & 0 & \multirow[t]{4}{*}{$00 \%$} & \multirow[t]{4}{*}{$00 \%$} & \multirow[t]{4}{*}{$00 \%$} & \multirow[t]{4}{*}{$16,7 \%$} \\
\hline & CASI SIEMPRE & 0 & & & & \\
\hline & A VECES & 0 & & & & \\
\hline & NUNCA & 1 & & & & \\
\hline \multirow{4}{*}{$\begin{array}{c}\text { BELLO } \\
\text { HORIZONTE }\end{array}$} & SIEMPRE & 0 & \multirow[t]{4}{*}{$00 \%$} & \multirow[t]{4}{*}{$00 \%$} & \multirow[t]{4}{*}{$00 \%$} & \multirow[t]{4}{*}{$16,7 \%$} \\
\hline & CASI SIEMPRE & 0 & & & & \\
\hline & A VECES & 0 & & & & \\
\hline & NUNCA & 1 & & & & \\
\hline \multirow{4}{*}{ RICARDO ORTIZ } & SIEMPRE & 0 & \multirow[t]{4}{*}{$00 \%$} & \multirow[t]{4}{*}{$00 \%$} & \multirow[t]{4}{*}{$00 \%$} & \multirow[t]{4}{*}{$33,3 \%$} \\
\hline & CASI SIEMPRE & 0 & & & & \\
\hline & A VECES & 0 & & & & \\
\hline & NUNCA & 2 & & & & \\
\hline \multicolumn{2}{|c|}{ TOTAL } & 6 & $00 \%$ & $00 \%$ & $00 \%$ & $100 \%$ \\
\hline
\end{tabular}

Fuente: Instituciones Distrito 9. Elaborada por los autores

En la Tabla IV, se puede decir que las docentes no cuentan con material relacionado a género, demostrando así que el material utilizado es reproductor de estereotipos de género ejemplo, si estamos trabajando una clase de profesiones, el material utilizado es el típico donde el niño será el doctor y la niña la enfermera o el niño policía y la niña costurera, este material no aporta el trabajo de género, se podría decir que en un futuro cercano no existan mujeres doctoras, ni policías como tampoco hombres enfermeros, sastres otros. 


\section{V.CONCLUSIONES}

A partir de los resultados obtenidos en la presente investigación, podemos conocer que en la práctica docente la manifestación de estereotipos de género se da en normas, reglas, códigos de comunicación, convivencia $\mathrm{y}$ actividades que supuestamente son solo para niños y para niñas, creadas a lo largo de la historia mediante la cultura y la sociedad [9]. Estás se manifiestan de manera implícita en el desarrollo de actividades, propiciando la competitividad más que la cooperación poniendo en juego modelos preestablecidos. Se puede concluir que se siguen desarrollándose estereotipos de género impartidos culturalmente en estos espacios.

Sobre los materiales y los recursos didácticos que se utilizan en el aula se encontró que presentan generalmente un sesgo sexista. Es necesario motivar a las docentes a la elaboración de nuevas imágenes y materiales cercanas a la realidad, esto posibilitará el reconocimiento de la activa participación de las mujeres en los ámbitos laboral, político y económico, conllevando a una cooperación con las mismas oportunidades para niños y niñas.

Construir una igualdad de género no se reduce a sustituir una letra por otra, es el hecho de considerar las diferencias, hacer visible lo que se ha invisibilizado por tantos años, se sugiere considerar las limitaciones que tiene el género femenino como el masculino, ya que a lo lar $\neg$ go de la revisión bibliográfica se ha encontrado cierta inclinación hacia la problemática de lo femenino, des $\neg$ virtuándose el sentido en la búsqueda de la igualdad; es por esto, que se motiva a realizar más estudios sobre los estereotipos de ambos géneros y sus conse $\neg-$ cuencias en nuestro contexto. Finalmente se sugiere investigar las expresiones lúdicas, ya que es ahí donde los infantes asumen las diferencias sexuales y genéricas.

\section{REFERENCIAS}

[1]G. Hernández Mendez, «Habitus, estereotipos y roles de género. Percepciones de profesores y estudiantes,» Docencia Universitaria, vol. 14, nº 1, pp. 89-105, diciembre 2003.

[2]E. Valencia Medina, «La evaluación de la calidad educativa para el mejoramiento del perfil profesional de educación,» Universidad Ciencia y Tecnologia, vol. 2, $\mathrm{n}^{\mathrm{o}} 2$, pp. 19-25, noviembre 2019.

[3]C. Salinas Boldo, «Equidad de géneros como unidad de aprendizaje en la universidad,» Alteridad, vol. 13, $\mathrm{n}^{\circ}$ 2, pp. 180-191, Julio 2018.

[4]M. d. Educación, Currriculo de educación Inicial, Quito: Ministerio de Educación, 2014.

[5]A. Constituyente, Constitución de la Republica del Ecuador, Quito: Ministerio del Ecuador, 2008, p. 27.
[6]O. B. Reyes Meza, F. M. Ávila Rosales, M. V. Andrade Torres y D. M. d. R. Alcívar Cedeño, «Influencia del género en los estilos de aprendizaje,» Universidad Ciencia y Tecnología, vol. 23, nº 94, pp. 48-53, Octubre 2019.

[7]H. Lia y M. Gracia, «Uso de la aplicación digital avaloe-ssd como instrumento de desarrollo docente para mejorar las competencias comunicativas y linguisticas de los alumnos de educación inicial,» Universidad Ciencia y Tecnología, vol. $2, \mathrm{n}^{\circ} 2$, pp. 39-44, Noviembre 2019.

[8]C. J. Quiaragua González, «¿Por qué los profesores guardan silencio acerca de la desigualdad de género en las escuelas?,» Actualidades investigativas en Educación, vol. 16, n⿳⺈ 3, pp. 1-21, Diciembre 2016.

[9]L. Camacho Brown y H. Watson Soto, «Reflexiones sobre Equidad de Género y Educación Inicial,» Inter Sedes, vol. VIII, $n^{\circ} 14$, pp. 33-48, julio 2007.

[10] L. Raspall, Lo que necesitan1@s niñ@as. ¿Una nueva escuela?, Rosario: HomoSapiens, 2019, pp. 37,121-135.

[11]A. E. Pozo Potosi, Enfoque de Género en el Curriculo de Educación Inicial de niños y niñas 3 a 4 años del distrito 9,Circuito 1, parroquia "El Quinche" del D.M. de Quito periodo 2014-2015; previo a optar el título de Magister, Quito: Facultad de Filosofía, Ciencias y Letras de la Educación. Universidad Central del Ecuador, 2014.

[12]M. J. Logroño, G. Borja Naranjo y C. P. Orozco-Ocaña, «Visión crítica del currículo desde el enfoque de género e interseccionalidad: Caso de la Universidad Central del Ecuador,» Entorno, Universidad Tecnológica de El Salvador, $n^{\circ}$ 66, pp. 237-255, Diciembre 2018. [13]E. Morin, Articular los saberes ¿Qué saberes enseñar en las escuelas?, Buenos Aires: Universisdad del Salvador, 2007, p. 34.

[14]M. F. Del Río, K. Strasser y M. I. Susperreguy, «Son las habilidades matemáticas un asunto de Género? Los estereotipos de género acerca de las matemáticas en niños y niñas de Kínder, sus familias y educadoras,» Calidad en la Educación, $n^{\circ} 45$, pp. 20-53, Diciembre 2016.

[15]J. Huamani Cahua, A. Serruto Castillo, V. A. Rivera Flores y C. I. Aguilar Del Carpio, «Actitud hacia la igualdad de género: Un estudio comparativo en adolecentes de la ciudad de Arequípa, Perú,» Universidad Ciencia y Tecnologia, vol. 24, $n^{\circ}$ 99, pp. 17-23, Abril 2020.

[16]S. Cueto, G. Espinosa, Y. Rodrígeuz, S. Giselle, G. Guerrero, J. León y C. Ramírez, «Educación, procesoso pedagógicos y equidad: cuatro informes de investigación,» Grupo de Análsis para el Desarrollo, GRADE, 
Lima-Perú, 2004.

[17]G. Mendez, «Habitus, estereotipos y roles de géne-

\section{RESUMEN CURRICULAR}

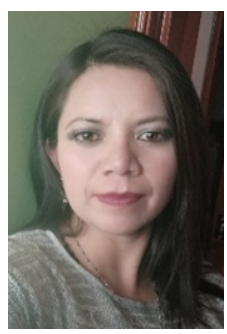

Pozo Potosi, Andrea Elizabeth; Magister en Educación y Proyectos de Desarrollo con Enfoque de Género, Licenciada en Ciencias de la Educación mención Profesora Parvularia, de nacionalidad ecuatoriana, docente del nivel Inicial en el Ministerio de Educación, apasionada de la lectura y la investigación. Cursando el Doctorado en Ciencias de la Educación en la Universidad Nacional de Rosario - Argentina.

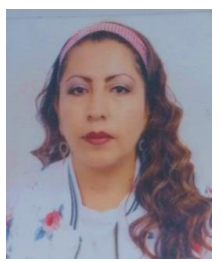

García, Ruth; Magister en Pedagogía de nacionalidad ecuatoriana, en la actualidad directivo de una institución educativa emblemática del sistema educativo fiscal gracias a su conocimiento y título en licenciatura con mención en gerencia y liderazgo educativo, amante de la lectura e investigación de las neurociencias y procesos educativos, razón por la cual su título inaugural lo obtuvo en el instituto superior Normal Manuela Cañizares con alusión en docencia de educación básica. En este momento se encuentra cursando el Doctorado en Ciencias de la Educación en la Universidad Nacional de Rosario Argentina. ro. Percepciones de profesores y estudiantes,» Docencia Universitaria, vol. 14, nº 1, pp. 89-105, 2014.

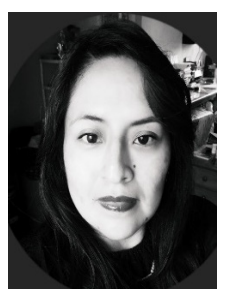

Casa Llano Erika Silvia; Magister en Educación Inicial, Licenciada en Ciencias de la Educación mención Profesora Parvularia, nacionalidad ecuatoriana, en la actualidad directivo de un Centro de Desarrollo Para la Primera Infancia Ministerio de Educación, su amor por la enseñanza en la educación Inicial, le ha motivado día a día para buscar siempre cambios e innovación en su área. Actualmente se encuentra cursando un Doctorado en Ciencias de la Educación en la Universidad Nacional de Rosario Argentina. https:/casa-erika.com/

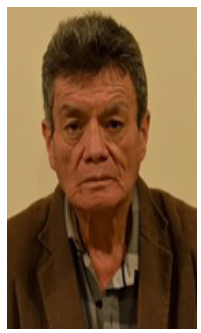

Logroño Galárraga, Moisés Rosendo; Magíster en Educación Superior; Especialista en Procesos Educativos; Docente Investigador de la Universidad Central del Ecuador; Coordinador de la Red Nacional de Educación Inicial, REI; Facilitador de Posgrados en varias universidades; Colaborador de la Propuesta Educativa Intercultural "Kintiku Yachay"; Autor de siete libros. 